\title{
OBSERVATIONAL EVIDENCE FOR EVOLUTIONARY CONNECTIONS BETWEEN WOLF-RAYET STARS AND RED SUPERGIANTS
}

\author{
Leonid N. Georgiev \\ Department of Astronomy \\ Sofia University \\ 5 Anton Ivanov Str. \\ Sofia 1126, Bulgaria.
}

The main question in our understanding of the nature of WR stars is: which stars are their progenitors and how do they evolve to reach the WR stage? We have some ideas about progenitor's masses and, by mass luminosity relations, about their brightness when they are $\mathrm{O}$ stars. But, it is difficult to say anything for their possible Red phase.

We suggest one statistical approach to this problem, based on the recent data for M33.

We have used the data for WR stars from Massey and Conti (1983), Armandroff and Massey (1985) and Massey et al. (1987). The stars with coincident coordinates ( $3^{\prime \prime}$ error box) and the same spectral index are excluded. The data for red and blue supergiants are taken from the catalog of Freedman (1984), appended by Ivanov (1990). The combined catalog is complete up to $19^{\mathrm{m}} .5$. Because we are interested in supergiants only, we stop our calculations at this limit. We have accepted as blue supergiants (BSG) the stars with $U-V<-0^{m} \cdot 3$, and for RSG these with $B-V>1^{m} .9$ (Humpheys et al., 1985).

The evolutionary theory predicts two main paths for stars with masses greater than $30 M_{\odot} \cdot$

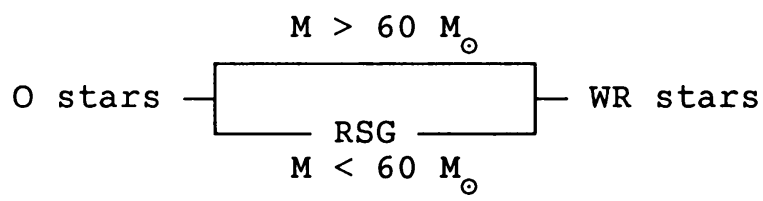

Thence the distribution of $\mathrm{O}$ stars $\left(\mathrm{P}_{B S G}\right)$ must be the same as the distribution of (RSG + WR) stars - $\mathrm{P}_{W R+R S G}$.

Assuming an apparent distance modulus to M33 of about $25^{m} .0$, we deal with $\mathrm{O}$ stars brighter than $\mathrm{M}_{v}=-5^{m} \cdot 5$, or with $M>30 M_{\odot}$. Also we have a fixed number of WR stars. So, to obtain a coincidence between the distributions mentioned above, we can vary only the magnitude $m_{1}$. RSG brighter than $m_{1}$ will be added to WR stars and the resulting distribution $\mathrm{P}_{W} R+R S G$ is compared with $\mathrm{P}_{B S G}$. The best coincidence occurs for $\mathrm{m}_{1}=$ $18^{m} .5$, and we conclude that the red stars in M33 brighter than $18^{m} .5$ evolve to WR stars.

Maeder et al. (1980) have shown that the lifetime of each phase depends on metal abundance. In favour of this result they suggest an observed gradient in relative numbers of WR stars $\left(\mathrm{N}_{W R}\right)$ and RSG $\left(\mathrm{N}_{R S G}\right)$. Using our limit magnitude $18^{m} .5$ for RSG we confirm the presence of a gradient of $\mathrm{N}_{W R} / \mathrm{N}_{R S G}$ in M33.

Based on the data of the chemical composition in M33 (Kwitter and Aller, 1981) we have also found that the ratio $\mathrm{N}_{W R} / \mathrm{N}_{R S G}$ is sensitive to the metallicity $\mathrm{Z}$.

References

Armandroff, T.E., and Massey, P., 1985, Astrophys. J. $291,685$.

Conti, P.S., and Massey, P., 1983, Astrophys. J. 273, 576.

Freedman, W.L., 1984 Thesis University of Toronto.

Humphreys, R.M., Nichols, M. and Massey, P., 1985 Astrophys. J. 90, 101.

Ivanov, G., 1990, in preparation.

Kwitter, K.B., and Aller, L.H., 1981 M.N.R.A.S. 195, 939.

Maeder, A., Lequeux, J. and Azzopardi, M., 1981, Astron. Astrophys. (Lett) 90, L17.

Massey, P, Conti, P., Moffat, A., and Shara, M., 1987 P.A.S.P. 99, 816.

K. A. van der Hucht and B. Hidayat (eds.),

Wolf-Rayet Stars and Interrelations with Other Massive Stars in Galaxies, 564.

(C) 1991 IAU. Printed in the Netherlands. 